\title{
Comprehensive pathway-related genes signature for prognosis and recurrence of ovarian cancer
}

\author{
Xinnan Zhao ${ }^{1}$, Miao He ${ }^{\text {Corresp. } 2}$ \\ ${ }^{1}$ Department of rheumatology and immunology, the first affiliated hospital of China Medical University, Shenyang, China \\ 2 Department of Pharmacology, China Medical University, Shenyang, China \\ Corresponding Author: Miao He \\ Email address: hemiao_cmu@163.com
}

Background Ovarian cancer $(\mathrm{OC})$ is a highly malignant disease with a poor prognosis and high recurrence rate. At present, there is no accurate strategy to predict the prognosis and recurrence of OC. The aim of this study was to identify gene-based signatures to predict OC prognosis and recurrence. Methods mRNA expression profiles and corresponding clinical information regarding OC were collected from The Cancer Genome Atlas (TCGA) database. Gene set enrichment analysis (GSEA) and LASSO analysis were performed, and Kaplan-Meier curves, time-dependent ROC curves, and nomograms were constructed using R software and GraphPad Prism7. Results We first identified several key signalling pathways that affected ovarian tumorigenesis by GSEA. We then established a 9-genebased signature for overall survival (OS) and a 5-gene-based-signature for relapse-free survival (RFS) using LASSO Cox regression analysis of the TCGA dataset and validated the prognostic value of these signatures in independent GEO datasets. We also confirmed that these signatures were independent risk factors for OS and RFS by multivariate Cox analysis. Time-dependent ROC analysis showed that the AUC values for OS and RFS were $0.640,0.663,0.758$, and 0.891 , and $0.638,0.722,0.813$, and 0.972 at $1,3,5$, and 10 years, respectively. The results of the nomogram analysis demonstrated that combining two signatures with the TNM staging system and tumour status yielded better predictive ability. Conclusion In conclusion, the two-gene-based signatures established in this study may serve as novel and independent prognostic indicators for OS and RFS. 
1 Comprehensive pathway-related genes signature for prognosis and recurrence of ovarian 2 cancer

3 Xinnan Zhao $^{1}$, Miao $\mathrm{He}^{2}$

$4{ }^{1}$ Department of rheumatology and immunology, the first affiliated hospital of China Medical 5 University

6 2Department of Pharmacology, China Medical University, No.77 Puhe Road, Shenyang North

7 New Area, Shenyang 110122, Liaoning Province, P. R. China

8 Xinnan Zhao E-mail: dr may@yeah.net

9 Miao He E-mail: hemiao_cmu@163.com

10 Short title: pathway-related signatures for OC

11 *Corresponding Author: Miao He, Department of Pharmacology, School of Pharmacy, China

12 Medical University, No.77 Puhe Road, Shenyang North New Area, Shenyang City 110122

13 Liaoning, China. E-mail: hemiao_cmu@163.com; Tel: +86/24/31939448; Fax: +86/24/31939448

14

15 


\section{Abstract}

\section{Background}

18 Ovarian cancer (OC) is a highly malignant disease with a poor prognosis and high recurrence rate. At present, there is no accurate strategy to predict the prognosis and recurrence of OC. The aim of this study was to identify gene-based signatures to predict OC prognosis and recurrence.

\section{1}

22

\section{Methods}

mRNA expression profiles and corresponding clinical information regarding OC were collected from The Cancer Genome Atlas (TCGA) database. Gene set enrichment analysis (GSEA) and LASSO analysis were performed, and Kaplan-Meier curves, time-dependent ROC curves, and nomograms were constructed using R software and GraphPad Prism7.

\section{Results}

We first identified several key signalling pathways that affected ovarian tumorigenesis by GSEA. We then established a 9-gene-based signature for overall survival (OS) and a 5-gene-basedsignature for relapse-free survival (RFS) using LASSO Cox regression analysis of the TCGA dataset and validated the prognostic value of these signatures in independent GEO datasets. We also confirmed that these signatures were independent risk factors for OS and RFS by multivariate Cox analysis. Time-dependent ROC analysis showed that the AUC values for OS and RFS were 0.640, 0.663, 0.758, and 0.891, and 0.638, 0.722, 0.813, and 0.972 at $1,3,5$, and 10 years, respectively. The results of the nomogram analysis demonstrated that combining two signatures with the TNM staging system and tumour status yielded better predictive ability.

\section{Conclusion}

In conclusion, the two-gene-based signatures established in this study may serve as novel and independent prognostic indicators for OS and RFS.

Keywords: Ovarian cancer, GSEA, LASSO, Overall survival, Recurrence-free survival

\section{INTRODUCTION}

Ovarian cancer (OC), one of the most common female malignant tumours, is the fifth-most 
42 common cause of mortality in women with severe gynaecological issues, with an estimated 13,940

43 deaths and 21,750 new cases reported in the 2020 United States cancer statistics (Siegel \& Miller

44 2020). Because OC is usually diagnosed at a late stage and currently lacks effective treatment

45 options, patients with OC have extremely poor prognosis and only a 30-40\%5-year survival rate

46 (EnrothBerggrundLyckeBrobergLundbergAssarssonOlovssonStalbergSundfeldt \& Gyllensten

47 2019). Despite the moderate improvements that have been made in the diagnosis and treatment of

48 OC over the past 30 years, OC is still a highly malignant disease that is life-threatening

49 (LiGuChenZhuZhaoWangZhaoZhangZhang \& Shu 2019). In addition, OC patients have an

50 average relapse-free survival (RFS) of two to three years after first-line therapy and die due to

51 chemotherapy resistance (DinhHarnettPiccart-Gebhart \& Awada 2008). Therefore, risk evaluation

52 involving prognosis and recurrence has critical value in clinical decisions and patient consultation.

53 To date, several common biomarkers for diagnosing OC remain ineffective due to lack of

54 sensitivity and specificity; these include carbohydrate antigen 125 (CA125, AUC $=0.630$ )

55 (ManshaGill \& Thomson 2019) and human epididymis protein 4 (HE4, AUC=0.719)

56 (ScalettaPlottiLuveroCapriglioneMonteraMirandaLopezTerranovaDe Cicco Nardone \& Angioli

57 2017). At the same time, a number of studies have identified certain genes that are significantly

58 associated with OC patient prognosis, such as TRIM44 (WangJiaHe \& Liu 2018) and CENPK

59 (LeeHuangLinChang \& Lee 2015). However, there is very limited prognostic value in a single

60 candidate biomarker, which is attributed to inconsistent sample collection, detection methods, and

61 small sample sizes. A number of studies have shown that combined biomarkers could improve

62 prognostic accuracy compared to single biomarkers (Barata \& Rini 2017). Thus, extensive studies

63 have attempted to establish molecular signatures based on gene expression to predict survival of

64 patients, including mRNA- (ZhouYiWangSu \& Luo 2019), microRNA-

65 (LiGuChenZhuZhaoWangZhaoZhangZhang \& Shu 2019), and long non-coding RNA-based

66 signatures (XuWuCheZhaoWangWangQuLiu \& Li 2019). However, only a small number of

67 prognostic signatures have been developed, and none have been directly applied in clinical 
68 practice. Hence, there is an urgent need to identify and validate novel, highly sensitive, and specific

69 molecular biomarkers for prognosis, monitoring, and therapy improvement.

70 In this study, we sought to explore signalling pathways related to ovarian tumorigenesis and

71 attempted to identify the potential predictive abilities of genes participating in these pathways for

72 OC prognosis and recurrence. We established a 9-gene signature panel for overall survival (OS)

73 and a 5-gene signature group for RFS using LASSO Cox regression analysis. Both signatures have

74 powerful prognostic predictive abilities and high sensitivity and specificity. Our results revealed

75 that both signatures were independent risk factors for OC survival and recurrence. In addition, we

76 constructed two nomogram models encompassing signature, TNM stage, and tumour status for OS

77 and RFS, respectively, and found that both nomogram models exhibited high potential clinical

78 utility by decision curve analysis (DCA). In general, we constructed two reliable and superior gene

79 signature panels as prognostic predictors of OC patient survival and recurrence.

80 2. MATERIALS AND METHODS

$81 \quad 2.1$ Data collection

82 The Cancer Genome Atlas (TCGA) -OC expression profiles (HTSeq-Counts) of genes and 83 corresponding clinical information were downloaded from the TCGA database

84 (http://cancergenome.nih.gov) (TomczakCzerwinska \& Wiznerowicz 2015). Detailed information

85 is presented in Table S1. Other datasets, including GSE40595, GSE12470, GSE10971, GSE27651

86 GSE38666, and GSE17260, were downloaded from the GEO database

87 (https://www.ncbi.nlm.nih.gov/geo/). Detailed information is shown in Table S2-S3.

$88 \quad 2.2$ Gene set enrichment analysis

89 Gene set enrichment analysis (GSEA) (http://www.broadinstitue.org/gsea/index.jsp) was

90 implemented using the JAVA.8 program using the Molecular Signatures Database

91 (SubramanianTamayoMoothaMukherjeeEbertGillettePaulovichPomeroyGolubLander \& Mesirov

92 2005). The hallmark gene sets were considered as a reference gene set and $P<0.05$ was considered

93 statistically significant. 
94

111 Nomograms can be used to predict the survival rates in diseases with multiple indicators

112 (IasonosSchragRaj \& Panageas 2008). To determine the clinical application of gene signatures,

113 we generated an optimal nomogram model using the "rms", "nomogramEx" and "regplot" R

114 packages to evaluate the 1-, 3- and 5-year OS and RFS of OC patients. DCA was performed to

115 assess the clinical usefulness of the risk prediction nomogram (Vickers \& Elkin 2006). The $\mathrm{x}$-axis

116 represented the percentage of threshold probability, and the y-axis represented the net benefit.

\section{$117 \quad 2.6$ Transcription factor analysis and GO enrichment analysis}

118 Transcription factor analysis was produced using a website 
120 implemented to further explore the biological function of Transcription factor. $P<0.05$ was

121 considered a significant enrichment.

\section{$122 \quad 2.7$ Statistical analysis}

123 OS and RFS differences between patients in high-risk and low-risk groups were estimated by

124 Kaplan-Meier survival curve analysis and calculated using log-rank tests. Time-dependent receiver

125 operating characteristic (time-dependent ROC) curves were constructed to determine the

126 sensitivity and specificity of gene signatures by calculating the area under curve (AUC) using the

127 "survivalROC" R package (DoLe \& Le 2020; Le 2019; LeYappNagasundaramChua \& Yeh 2019).

128 We compared two groups using Student's $t$-test for numerical variables and Chi-squared tests for

129 categorical variables. All statistical graphs were produced using R (https:/www.r-project.org/,

130 v3.5.1), SPSS v.22.0 (SPSS Inc., Chicago, IL) and GraphPad Prism7 (GraphPad Software Inc., La

131 Jolla, CA).

\section{3. RESULTS}

\section{3.1 Identification of signalling pathways correlated with OC tumorigenesis}

134 To determine related signalling pathways involved in ovarian tumorigenesis, we performed GSEA

135 on Hallmark gene sets for five independent GEO datasets, GSE40595, GSE12470, GSE10971,

136 GSE27651, and GSE38666. The results showed that there are multiple signalling pathways

137 significantly enriched in OC including "MYC_TARGETS_V1", "MTORC1_SIGNALING",

138 "GLYCOLYSIS", "E2F_TARGETS", "G2M_CHECKPOINT",

139 "OXIDATIVE_PHOSPHORYLATION", "DNA_REPAIR" and

140 "PI3K_AKT_MTOR_SIGNALING" (Figure 1A-E and Table S4), suggesting that these signalling

141 pathways might play critical roles in the development of OC.

\section{3.2 Establishment of gene signatures for overall survival and relapse-free survival}

143 To evaluate the effect of genes in these pathways on OS and RFS, we performed univariate Cox

144 regression analysis using TCGA data. The results showed that 48 and 44 genes were correlated 145 with patient OS and RFS, respectively. Furthermore, the LASSO Cox selection method was 
146 implemented to establish a prognosis-predicting model (OS: Figure 2A, B; RFS: Figure 2C, D).

147 Finally, a 9-gene signature panel for OS and a 5-gene signature group for RFS were established

148 by LASSO Cox regression analysis. The formula of the 9-gene signature panel for OS $=(-0.0027)$

$149 *$ expression of $H M G B 3+0.0122 *$ expression of $P Y G B+0.0094 *$ expression of $I D U A+0.0092$

$150 *$ expression of $L H X 9+(-0.0254) *$ expression of SLC7A11 + $(-0.0037) *$ expression of

151 DPYSL4 $+0.0017 *$ expression of ANGPTL4 $+(-0.0357) *$ expression of ISG20 $+(-0.0353) *$

152 expression of GRM8; and the formula of the 5-gene signature group for RFS $=(-0.0114) *$

153 expression of SNRPA1 + (-0.0346) * expression of WARS + $0.0234 *$ expression of CITED2 +

$1540.002 *$ expression of ANGPTL4 $+0.0357 *$ expression of EFNA5 (Table S5). Next, we calculated

155 risk scores for the OS and RFS of each patient based on the above formula. The distribution of risk

156 scores, survival times, patient status, and expression heat maps of genes were obtained. The results

157 showed that patients in the high-risk group had shorter OS and RFS and higher mortality than

158 those in the low-risk group (Figure 3A, B and Figure S1A, B). According to these two signatures,

159 patients were divided into high-risk or low-risk groups using the median risk score as the cut-off

160 point (OS: median=0.863; RFS: median=0.457). We performed Kaplan-Meier analysis and found

161 that patients with high risk scores had significantly shorter OS and RFS than those with low risk

162 scores $(\mathrm{OS}: P<0.0001, \mathrm{HR}=2.289,95 \% \mathrm{CI}=1.724-3.04$; RFS: $P<0.0001, \mathrm{HR}=1.783,95 \% \mathrm{CI}$

$163=1.465-2.264$; Figure 4A, B). Furthermore, to explore the sensitivity and specificity of the risk

164 score, we performed time-dependent ROC analysis. The results showed that for OS, the prognostic

165 accuracy of the 9-gene signature panel was $0.640,0.663,0.758$, and 0.891 at $1,3,5$, and 10 years,

166 respectively. The AUCs for RFS were $0.638,0.722,0.813$, and 0.972 at 1,3 , 5, and 10 years,

167 respectively (Figure 4C, D). These results demonstrate that the 9-gene signature panel is a helpful

168 indicator of OS for OC patients and that the 5-gene signature group is a powerful prognostic

169 predictor of OC patient recurrence.

$170 \quad 3.3$ Validation of the 9-gene signature panel for overall survival and 5-gene signature group 171 for relapse-free survival 
172 To verify the prognostic value of our signatures, we investigated the independent dataset

173 GSE17260 for validation of OS and RFS. We also performed Kaplan-Meier analysis and observed

174 the same results as above, which showed that patients in the high-risk group had shorter OS and

175 RFS and higher mortality than those in the low-risk group based on our 9- and 5-gene-based

176 signatures, respectively (Figure 5A, B). In addition, we performed cross validation to prove the

177 consistency of these signatures. We found the same gene sets in GSE17260 dataset (Figure S2A,

178 B), the only difference is the coefficients of each gene have changed a little. Furthermore, we also

179 performed Kaplan-Meier analysis and observed the same results in TCGA dataset (Figure S2C,

180 D).

$181 \quad 3.4$ The signature perform better in survival prediction than other signature

182 Then, the ROC curves were implemented to contrast prognostic ability between our signature and 183 other signature (risk score $=0.38 *$ XPC $-0.24 *$ PALB2 $+0.29 *$ RECQL $-0.18 *$ XRCC2 + $1840.32 *$ GTF2H5 - 0.19*GTF2H4 - 0.22*SSBP1 + 0.24*RAD54L - 0.25*MUTYH - 0.3*SMUG1 $\left.185-0.16^{*} \mathrm{TDP} 1+0.24 * \mathrm{DDB} 2+0.26^{*} \mathrm{RNH} 1+0.18^{*} \mathrm{TP} 53 \mathrm{BP} 1\right)$

186 (SunCaoMaYangPengYuZhouZhangLiHuo \& Shen 2019). The result of ROC analysis illustrated 187 that our signature has significantly higher ROC (AUC $=0.891)$ than other signature $(\mathrm{AUC}=0.751)$ 188 (Figure S3). This result demonstrated that predictive power of our signature is greater than other 189 signature. In general, our signature could be used as a superior indicator to predict the prognosis 190 of OV patients.

$191 \quad 3.5$ Relationships involving gene signatures and clinical characteristics

192 To further investigate potential correlations between risk scores and pat_ient clinical 193 characteristics, we conducted Chi-squared tests. We found that for OS, the risk score was

194 significantly associated with race and tumour status. Similarly, for RFS, the risk score was 195 significantly correlated with TNM stage and tumour status. However, for both OS and RFS, there 196 was no significant correlation between risk score and other clinical characteristics, including age 197 and tumour grade (Tables 1 and 2). 
198 3.6 Prognostic value of gene signatures is independent of other clinical characteristics

199 To further evaluate the predictive ability of gene signatures and other clinical characteristics in 200 terms of OS and RFS, univariate and multivariate Cox analyses were performed. As shown in

201 Figure $6 \mathrm{~A}$ and $6 \mathrm{~B}$, the 9-gene signatures, stage, race, and tumour status were significantly 202 correlated with OS, as seen in the univariate Cox regression analysis. Furthermore, multivariate 203 Cox regression analysis results showed that the 9-gene signatures and tumour status were 204 independent prognostic factors for OS. Meanwhile, univariate Cox regression analysis revealed 205 that the 5-gene signatures, staging, and tumour status were significantly associated with RFS. The 206 5-gene signatures and tumour status were independent prognostic factors for RFS after 207 multivariate Cox regression analysis.

\section{$208 \quad 3.7$ Clinical application of gene signatures}

209 We further constructed a nomogram predictive model combining gene signatures, race, stage, and

210 tumour status to predict the probability of 1-, 3-, and 5-year OS and RFS in OC patients (Figure

211 7A and 7C). The results showed that the nomogram model combined gene signatures, stage, and

212 tumour status (OS: C-index: 0.743; RFS: C-index: 0.712). These results demonstrate that our gene

213 signature-based nomogram approach exhibits superior performance in clinical settings.

214 Furthermore, DCA was conducted to evaluate the clinical usefulness of the risk prediction

215 nomogram for OS and RFS (Figure 7B and 7D). The DCA results showed that for OS and RFS,

216 the complex factor had a broader range of threshold probabilities and higher net benefits than

217 single clinical characteristics or risk scores. Taken together, the DCA results demonstrate that our

218 nomogram for OS and RFS has high potential clinical utility.

\section{$219 \quad 3.8$ Transcription factor analysis and GO enrichment analysis}

220 Transcription factors are a family of DNA-binding proteins whose gene regulatory capabilities are 221 of vital importance in defining the molecular state of a cell. Therefore, we performed Transcription 222 factor analysis of genes using website

223 (https://www.gcbi.com.cn/gcanalyze/html/generadar/index). To explore the potential function of 
224 these transcription factor, we performed GO enrichment analysis by clusterprofiler package. The

225 GO analysis results showed that these transcription factor analysis were mainly enriched in "cell

226 fate commitment", "pattern specification process", "embryonic organ development" and so on

227 (Figure S4).

\section{4. DISCUSSION}

229 To a certain extent, indolent and aggressive cancers cannot be accurately stratified by the TNM

230 staging system, which mostly relies on anatomical information without any molecular biological

231 information or features (ChoudharySharmaSinghChishi \& Garg 2019;

232 McIntyreRambauChanYapMorrisNelson \& Kobel 2017). Therefore, it is indispensable to identify

233 novel and effective predictive biomarkers for OC prognosis. In this study, we established and

234 validated novel prognostic gene-based signatures for OS and RFS to improve the prediction of

235 survival and tumour recurrence in OC patients.

236 Increasing number of studies are gradually clarifying the key signalling pathways involved in

237 cancer initiation, progression, and metastasis, including the PI3K-Akt signalling pathway 238 (MabuchiKurodaTakahashi \& Sasano 2015), MYC signalling pathway (Dang 2012) and so on.

239 Moreover, the genes participating in these key signalling pathways might play critical roles in

240 tumorigenesis. Therefore, it is necessary to explore the functions and roles of these key genes.

241 Consistently, extensive studies have confirmed several signalling pathway-related genes that are

242 significantly associated with the prognosis of cancer patients. Cao et al. confirmed that EMT-

243 related genes promote bladder cancer subtype transition from non-muscle invasive bladder cancer

244 (NMIBC) to muscle invasive bladder cancer (MIBC) and affect the prognosis of bladder cancer

245 patients (CaoYuanMaWangQiu \& Tian 2019). In this study, our candidate genes for OS and RFS

246 were screened for signalling pathways affecting the development of OC, including 247 "MYC_TARGETS_V1", "MTORC1_SIGNALING", "GLYCOLYSIS", "E2F_TARGETS" and 248 "G2M_CHECKPOINT". MYC is one of the most highly amplified oncogenes in many human 249 cancers and plays a pivotal role in tumorigenesis (Dang 2012). Mammalian target of rapamycin 
250 complex 1 (mTORC1) signalling is found to be elevated in many human cancers, including OC,

251 and promotes cell growth, proliferation, and metabolism (Menon \& Manning 2008). Increasing

252 evidence suggests that aerobic glycolysis is related to tumour growth and chemotherapy resistance

253 (ChakrabortyMustafiXiongDwivediNesinSahaZhangDhanasekaran \& Jayaraman 2017). The E2F

254 family plays a significant role in cancer initiation, progression, and resistance to therapy (Kent \&

255 Leone 2019) and E2Fs can mediate fundamental cell-cycle deregulation in high-grade serous OC

256 (De MeyerBijsmansVan de VijverBekaertOostingVan Criekingevan Engeland \& Sieben 2009).

257 Hence, it is a plausible inference for these genes to be associated with the survival of patients with

258 clear cell renal cell carcinoma (ccRCC).

259 High-dimensional data survival models have a high risk of overfitting, which is characterised by

260 reduced importance when applying predictors to independent datasets. To solve this problem, we

261 applied a Cox proportional hazard model using the LASSO penalty method to optimise gene

262 selection. This is useful for selecting genes with strong prognostic value, high expression

263 differences, and low correlation among each other (Tibshirani 1997). The potential of LASSO has

264 been previously implemented in colon cancer (DaiLiMoFengZhangXuLi \& Cai 2018), bladder

265 cancer (HeHePengZhanLiChenGongLi \& Zhou 2019) and other studies. Therefore, in this study,

266 we successfully applied this method and established a 9-gene-based signature panel for OS and a

267 5-gene-based signature group for RFS to optimise the predictive ability of prognosis in OC

268 patients.

269 Although extensive studies have advocated several molecular biomarkers for risk stratification in

270 OC, along with traditional clinicopathologic parameters, there are still some points to be improved

271 as follows: (1) small sample sizes need to be enlarged; (2) independent validation datasets are

272 needed to further confirm the stability and accuracy of the established signatures; (3) it is

273 indispensable to compare the predictive power between signatures and the existing TNM staging

274 system; and (4) it is more valuable to explore prognostic biomarkers for OC recurrence. In our

275 study, we established novel superior prognostic signatures for OS and RFS in the TCGA dataset 
276 and validated the prognostic value of these signatures using an independent GEO dataset,

277 suggesting the repeatability and practicability of our two signatures. Then, we confirmed that these

278 signatures were independent risk factors for OS and RFS by univariate and multivariate Cox

279 analyses. In addition, we constructed a nomogram model to better predict survival of OC patients

280 and validated its promising clinical application by DCA. In general, our findings provided a

281 superior and useful biomarker for OS and RFS in OC patients, based on the results of the LASSO

282 regression analysis.

283 Our study demonstrated that 9-gene- and 5-gene-based signatures for OS and RFS, respectively,

284 were both closely associated with the prognosis of OC patients. However, the functions of most of

285 the genes in our signatures have not been entirely elucidated. For genes associated with OS, the

286 chromatin-associated high-mobility group box 3 (HMGB3) protein is upregulated and has poor

287 prognosis, and its targeted depletion can attenuate cisplatin resistance in human OC cells

288 (MukherjeeHuynhGainesReh \& Vasquez 2019). PYGB has been shown to be upregulated in OC

289 tissue and markedly associated with poor prognosis in OC patients (ZhouJin \& Wang 2019). LHX9

290 is a biomarker in ovarian stem cells (Auersperg 2013). SLC7A11 is an independent prognostic risk

291 factor for OS in OC (YinYiWeiZhaoLiCaiDong \& Liu 2019). Low levels of DPYSL4 are

292 significantly correlated with poor prognosis in OC

293 (NaganoHashimotoNakayamaSuzukiMiyabayashiYamatoHiguchiFujimotoSakumaBeppuYokoy

294 amaSuzukiSuganoIkedaTatsunoManabeYokoteInoue \& Tanaka 2018). In addition, Jiang et al.

295 suggested that DPYSL4 is significantly associated with OS in hepatocellular carcinoma

296 (JiangZhaoBiGuanQiWeiHeWei \& Zhao 2019). High expression of ISG20 is related to poor

297 clinical outcomes in glioblastoma (GaoWangWangWangZhang \& Sun 2019). GRM8 is a tumour-

298 suppressor gene in endometrial cancer cell lines (LiangCheungLiJuYuStemke-

299 HaleDogrulukLuLiuGuGuoSchererCarterWestinDyerVerhaakZhangKarchinLiuLuBroaddusScot

300 tHennessy \& Mills 2012). For genes associated with RFS, SNRPA1 is an oncogene in colorectal

301 cancer (ZengLeiChangGaoWangGaoNiu \& Li 2019). Yanagie et al. demonstrated that CBP/p300- 
302 interacting transactivator (CITED2) is potentially a multidrug-resistance gene, which is consistent

303 with

our

results

304 (YanagieHisaOgataMiyazakiNonakaNishihiraOsadaSairennjiSugiyamaFuruyaKidaniTakamotoT

305 akahashi \& Eriguchi 2009). ANGPTL4 is a novel biomarker of hypoxia in OC, and is upregulated

306 in partial responders to chemotherapy compared to responders

307 (McEvoyO'TooleSpillaneMartinGallagherStordalBlackshieldsSheils \& O'Leary 2015). Yang et

308 al. found that EFNA5 can be used as a novel prognostic biomarker and potential therapeutic target

309 for OC patients (YangBieSun \& Yue 2019).

310 Inevitably, there are certain innate limitations to our study that need to be addressed. First,

311 screening for these signatures was based on data from TCGA and GEO datasets without validating

312 their prognostic value in clinical practice. Second, although these candidate genes are involved in

313 key signalling pathways that influence prognosis in $\mathrm{OC}$, the mechanisms underlying some genes

314 in our signature panels remains unclear. Undoubtedly, further investigation of the roles of these

315 genes in OC progression is needed. It is reported that blacks vs whites were over two-fold less

316 likely to receive a surgery-chemotherapy, suggesting that race may affect survival status of ovarian

317 cancer patients greatly, which was consistent with our results. In addition, higher socioeconomic

318 status is associated with a greater probability of undergoing surgical resection and with improved

319 survival in patients with ovarian cancer, demonstrating socioeconomic status might also affect

320 prognosis of patients. Unfortunately, several important clinical characteristics, such as treatment

321 modality and socioeconomic status are not available in the TCGA cohort. Finally, prospective,

322 large-scale, multicentre studies are indispensable to verify the prognostic ability of our signatures

323 before applying them in clinical settings.

\section{5. CONCLUSIONS}

325 In conclusion, we established a 9-gene-based signature panel for OS, and a 5-gene-based signature

326 group for RFS using a LASSO Cox regression model. These signatures could be potentially novel

327 and independent prognostic indicators for predicting survival and disease recurrence in OC 
328 patients. Moreover, combining these signatures with current clinical indicators may serve to

329 optimise their predictive ability and guide future clinical planning for patient monitoring.

\section{Author contribution statement}

$331 \mathrm{XZ}$ and $\mathrm{MH}$ designed the study. XZ performed research and analyzed the data. XZ and MH wrote

332 the first draft of the paper, and $\mathrm{XZ}$ and $\mathrm{MH}$ edited the manuscript.

\section{Acknowledgments}

334 Not applicable.

\section{Funding Statement}

336 Not applicable.

\section{Conflict of Interest Statement}

338 The authors declare that there is no conflict of interest.

\section{Data Availability Statements}

340 The gene expression data and corresponding clinical information can be download from TCGA

341 database (http://cancergenome.nih.gov) and GEO database (https://www.ncbi.nlm.nih.gov/geo/).

\section{Reference}

Auersperg N. 2013. The stem-cell profile of ovarian surface epithelium is reproduced in the oviductal fimbriae, with increased stem-cell marker density in distal parts of the fimbriae. Int J Gynecol Pathol 32:444-453.

Barata PC, and Rini BI. 2017. Treatment of renal cell carcinoma: Current status and future directions. CA Cancer J Clin 67:507-524.

Cao R, Yuan L, Ma B, Wang G, Qiu W, and Tian Y. 2019. An EMT-related gene signature for the prognosis of human bladder cancer.

Chakraborty PK, Mustafi SB, Xiong X, Dwivedi SKD, Nesin V, Saha S, Zhang M, Dhanasekaran D, and Jayaraman M. 2017. MICU1 drives glycolysis and chemoresistance in ovarian cancer. 8:14634.

Choudhary D, Sharma G, Singh T, Chishi N, and Garg PK. 2019. Isolated abdominal wall metastasis in early ovarian cancer: a true systemic metastasis or local tumour cells implantation. J Obstet Gynaecol 39:724-726.

Dai W, Li Y, Mo S, Feng Y, Zhang L, Xu Y, Li Q, and Cai G. 2018. A robust gene signature for the prediction of early relapse in stage I-III colon cancer. Mol Oncol 12:463-475.

Dang CV. 2012. MYC on the path to cancer. Cell 149:22-35.

De Meyer T, Bijsmans IT, Van de Vijver KK, Bekaert S, Oosting J, Van Criekinge W, van Engeland M, and Sieben NL. 2009. E2Fs mediate a fundamental cell-cycle deregulation in high-grade serous ovarian carcinomas. J Pathol 217:14-20. 
360

361

362

363

364

365

366

367

368

369

370

371

372

373

374

375

376

377

378

379

380

381

382

383

384

385

386

387

388

389

390

391

392

393

394

395

396

397

398

Dinh P, Harnett P, Piccart-Gebhart MJ, and Awada A. 2008. New therapies for ovarian cancer: cytotoxics and molecularly targeted agents. Crit Rev Oncol Hematol 67:103-112.

Do DT, Le TQT, and Le NQK. 2020. Using deep neural networks and biological subwords to detect protein Ssulfenylation sites. Brief Bioinform.

Enroth S, Berggrund M, Lycke M, Broberg J, Lundberg M, Assarsson E, Olovsson M, Stalberg K, Sundfeldt K, and Gyllensten U. 2019. High throughput proteomics identifies a high-accuracy 11 plasma protein biomarker signature for ovarian cancer. Commun Biol 2:221.

Gao Y, Wang Y, Wang X, Wang Y, Zhang X, and Sun X. 2019. TNF-like ligand 1A is associated with progression and prognosis of human gastric cancer. Onco Targets Ther 12:7715-7723.

He A, He S, Peng D, Zhan Y, Li Y, Chen Z, Gong Y, Li X, and Zhou L. 2019. Prognostic value of long non-coding RNA signatures in bladder cancer. Aging (Albany NY) 11:6237-6251.

Iasonos A, Schrag D, Raj GV, and Panageas KS. 2008. How to build and interpret a nomogram for cancer prognosis. J Clin Oncol 26:1364-1370.

Jiang L, Zhao L, Bi J, Guan Q, Qi A, Wei Q, He M, Wei M, and Zhao L. 2019. Glycolysis gene expression profilings screen for prognostic risk signature of hepatocellular carcinoma. Aging (Albany NY) 11.

Kent LN, and Leone G. 2019. The broken cycle: E2F dysfunction in cancer. 19:326-338.

Le NQK. 2019. Fertility-GRU: Identifying Fertility-Related Proteins by Incorporating Deep-Gated Recurrent Units and Original Position-Specific Scoring Matrix Profiles. J Proteome Res 18:3503-3511.

Le NQK, Yapp EKY, Nagasundaram N, Chua MCH, and Yeh HY. 2019. Computational identification of vesicular transport proteins from sequences using deep gated recurrent units architecture. Comput Struct Biotechnol J 17:1245-1254.

Lee YC, Huang CC, Lin DY, Chang WC, and Lee KH. 2015. Overexpression of centromere protein K (CENPK) in ovarian cancer is correlated with poor patient survival and associated with predictive and prognostic relevance. PeerJ 3:e1386.

Li L, Gu H, Chen L, Zhu P, Zhao L, Wang Y, Zhao X, Zhang X, Zhang Y, and Shu P. 2019. Integrative Network Analysis Reveals a MicroRNA-Based Signature for Prognosis Prediction of Epithelial Ovarian Cancer. Biomed Res Int 2019:1056431.

Liang H, Cheung LW, Li J, Ju Z, Yu S, Stemke-Hale K, Dogruluk T, Lu Y, Liu X, Gu C, Guo W, Scherer SE, Carter H, Westin SN, Dyer MD, Verhaak RG, Zhang F, Karchin R, Liu CG, Lu KH, Russell R, Broaddus KL, Hennessy BT, Mills GB. 2012. Whole-exome sequencing combined with functional genomics reveals novel candidate driver cancer genes in endometrial cancer. Genome Res 22:2120-2129.

Mabuchi S, Kuroda H, Takahashi R, and Sasano T. 2015. The PI3K/AKT/mTOR pathway as a therapeutic target in ovarian cancer. Gynecol Oncol 137:173-179.

Mansha M, Gill A, and Thomson PC. 2019. Potential risk factors of ovarian cancer and analysis of CA125, a biomarker used for its monitoring and diagnosis. Mol Biol Rep 46:3325-3332.

McEvoy LM, O'Toole SA, Spillane CD, Martin CM, Gallagher MF, Stordal B, Blackshields G, Sheils O, and O'Leary JJ. 2015. Identifying novel hypoxia-associated markers of chemoresistance in ovarian cancer. BMC Cancer 15:547.

McIntyre JB, Rambau PF, Chan A, Yap S, Morris D, Nelson GS, and Kobel M. 2017. Molecular alterations in indolent,

Peer) reviewing PDF | (2020:07:50886:2:0:NEW 3 Nov 2020) 
aggressive and recurrent ovarian low-grade serous carcinoma. Histopathology 70:347-358.

Menon S, and Manning BD. 2008. Common corruption of the mTOR signaling network in human tumors. Oncogene 27 Suppl 2:S43-51.

Mukherjee A, Huynh V, Gaines K, Reh WA, and Vasquez KM. 2019. Targeting the High-Mobility Group Box 3 Protein Sensitizes Chemoresistant Ovarian Cancer Cells to Cisplatin. 79:3185-3191.

Nagano H, Hashimoto N, Nakayama A, Suzuki S, Miyabayashi Y, Yamato A, Higuchi S, Fujimoto M, Sakuma I, Beppu M, Yokoyama M, Suzuki Y, Sugano S, Ikeda K, Tatsuno I, Manabe I, Yokote K, Inoue S, Tanaka T. 2018. p53inducible DPYSL4 associates with mitochondrial supercomplexes and regulates energy metabolism in adipocytes and cancer cells. 115:8370-8375.

Scaletta G, Plotti F, Luvero D, Capriglione S, Montera R, Miranda A, Lopez S, Terranova C, De Cicco Nardone C, and Angioli R. 2017. The role of novel biomarker HE4 in the diagnosis, prognosis and follow-up of ovarian cancer: a systematic review. Expert Rev Anticancer Ther 17:827-839.

Siegel RL, and Miller KD. 2020. Cancer statistics, 2020. 70:7-30.

Subramanian A, Tamayo P, Mootha VK, Mukherjee S, Ebert BL, Gillette MA, Paulovich A, Pomeroy SL, Golub TR, Lander ES, Mesirov JP. 2005. Gene set enrichment analysis: a knowledge-based approach for interpreting genome-wide expression profiles. Proc Natl Acad Sci U S A 102:15545-15550.

Sun H, Cao D, Ma X, Yang J, Peng P, Yu M, Zhou H, Zhang Y, Li L, Huo X, Shen K. 2019. Identification of a Prognostic Signature Associated With DNA Repair Genes in Ovarian Cancer. Front Genet 10:839.

Tibshirani R. 1997. The lasso method for variable selection in the Cox model. Stat Med 16:385-395.

Tomczak K, Czerwinska P, and Wiznerowicz M. 2015. The Cancer Genome Atlas (TCGA): an immeasurable source of knowledge. Contemp Oncol (Pozn) 19:A68-77.

Vickers AJ, and Elkin EB. 2006. Decision curve analysis: a novel method for evaluating prediction models. Med Decis Making 26:565-574.

Wang S, Jia M, He Z, and Liu XS. 2018. APOBEC3B and APOBEC mutational signature as potential predictive markers for immunotherapy response in non-small cell lung cancer. Oncogene 37:3924-3936.

Xu L, Wu Y, Che X, Zhao J, Wang F, Wang P, Qu X, Liu Y, and Li Z. 2019. Cox-LASSO Analysis Reveals a Ten-IncRNA Signature to Predict Outcomes in Patients with High-Grade Serous Ovarian Cancer. DNA Cell Biol 38:15191528.

Yanagie H, Hisa T, Ogata A, Miyazaki A, Nonaka Y, Nishihira T, Osada I, Sairennji T, Sugiyama H, Furuya Y, Kidani Y, Takamoto S, Takahashi H, Eriguchi M. 2009. Improvement of sensitivity to platinum compound with siRNA knockdown of upregulated genes in platinum complex-resistant ovarian cancer cells in vitro. Biomed Pharmacother 63:553-560.

Yang L, Bie L, Sun L, and Yue Y. 2019. Neural activities are unfavorable for the prognosis of ovarian cancer through mRNA expression analysis. Biomark Med 13:663-673.

Yin F, Yi S, Wei L, Zhao B, Li J, Cai X, Dong C, and Liu X. 2019. Microarray-based identification of genes associated with prognosis and drug resistance in ovarian cancer. 120:6057-6070.

Zeng Q, Lei F, Chang Y, Gao Z, Wang Y, Gao Q, Niu P, and Li Q. 2019. An oncogenic gene, SNRPA1, regulates PIK3R1, VEGFC, MKI67, CDK1 and other genes in colorectal cancer. Biomed Pharmacother 117:109076.

Zhou J, Yi Y, Wang C, Su C, and Luo Y. 2019. Identification of a 3-mRNA signature as a novel potential prognostic 


\section{Figure legends}

443 Figure 1. The GSEA enrichment analysis of different GEO datasets. (A) GSE12470. (B) 444 GSE27651. (C) GSE38666. (D) GSE40595. (E) GSE10971.

445 Figure 2. The LASSO Cox regression model for OS and RFS of patients in TCGA dataset. The 446 LASSO coefficients profiles of prognosis-related genes for OS (A) and RFS (C). Tuning parameter

447 ( $\lambda$ ) selection cross-validation error curve for OS (B) and RFS (D). The number of lines above the 448 graph represent the number of genes in volved in the LASSO analysis, with the change of the Log 449 lambda, the most representative genes were selected.

450 Figure 3. Establishment of gene signature for OS and RFS in TCGA dataset. Risk score 451 distribution, patients' survival and status and expression heatmap of genes for OS (A) and RFS 452 (B).

453 Figure 4. Kaplan-Meier curve analysis and Time-dependent ROC curve analysis. Kaplan-Meier 454 curve analysis for OS (A) and RFS (B). Time-dependent ROC curve analysis of the sensitivity and 455 specificity for OS (C) and RFS (D).

456 Figure 5. Kaplan-Meier curve analysis. Kaplan-Meier curve analysis for OS (A) and RFS (B) in 457 GSE17260 dataset.

458 Figure 6. Prognostic value of gene signature is independent of other clinical characteristics. Forest 459 plot summary of univariate and multivariate Cox regression analysis of gene signature and clinical 460 characteristics on OS (A) and RFS (B).

461 Figure 7. Nomogram to predict 1-, 3- and 5-year OS and RFS for ovarian cancer patients. The 462 nomogram for predicting survival rate of patients with 1-, 3- and 5-year OS (A) and RFS (C) 463 (stage: $0=$ stage I, $1=$ stage II, $2=$ stage III, $3=$ stage IV; tumor status: $0=$ tumor free, $1=$ with 464 tumor). Decision analysis curve for OS (B) and RFS (D). 


\section{Supplementary Figure legend}

466 Figure S1. Risk score distribution in alive and dead patients for OS (A) and RFS (B).

467 Figure S2. Kaplan-Meier curve analysis. Kaplan-Meier curve analysis for OS (A, C) and RFS (B, 468 D) in GSE17260 dataset and TCGA dataset.

469 Figure S3. Comparison of time-dependent ROC analysis of our signature with other mRNA 470 signature.

471 Figure S4. GO enrichment analysis of transcription factor.

472 
Figure 1

The GSEA enrichment analysis of different GEO datasets.

The GSEA enrichment analysis of different GEO datasets. (A) GSE12470. (B) GSE27651. (C) GSE38666. (D) GSE40595. (E) GSE10971.

A

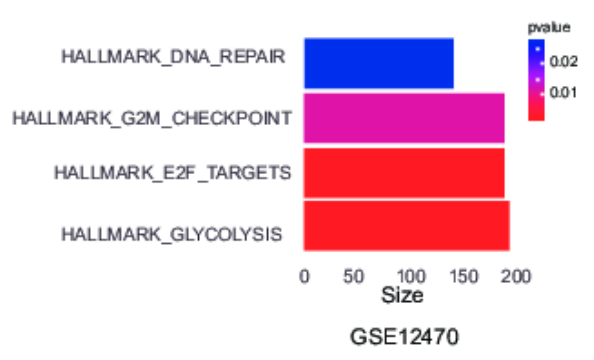

D

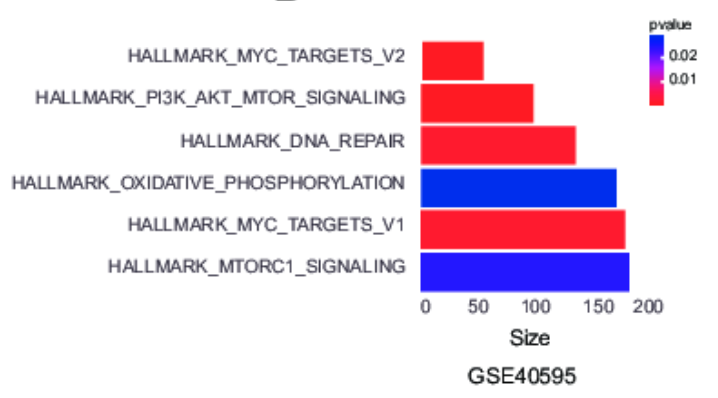

B

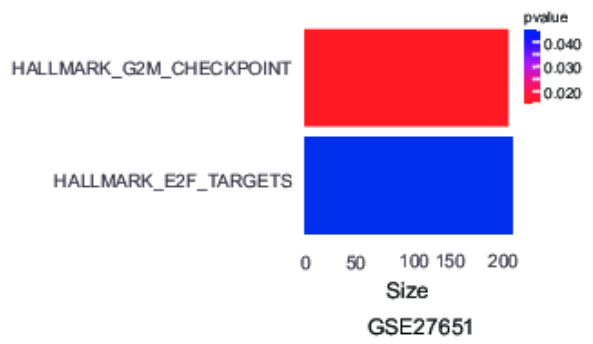

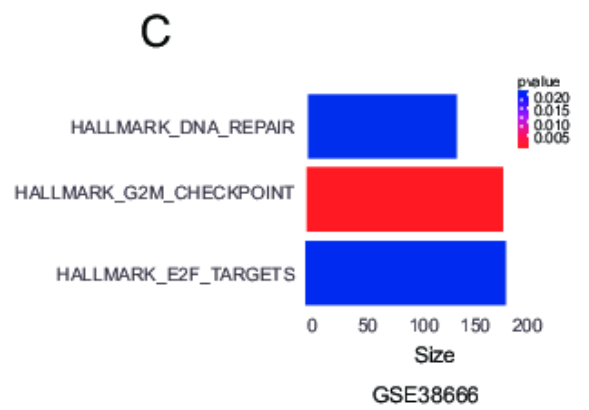

E

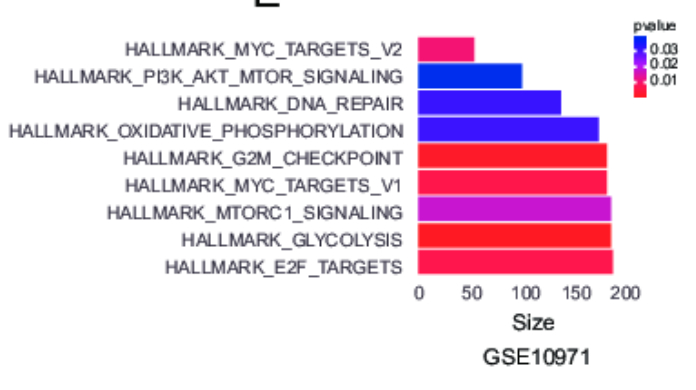




\section{Figure 2}

The LASSO Cox regression model for OS and RFS of patients in TCGA dataset.

The LASSO Cox regression model for OS and RFS of patients in TCGA dataset. The LASSO coefficients profiles of prognosis-related genes for OS (A) and RFS (C). Tuning parameter ( $\lambda$ ) selection cross-validation error curve for OS (B) and RFS (D). The number of lines above the graph represent the number of genes in volved in the LASSO analysis, with the change of the Log lambda, the most representative genes were selected. 
A

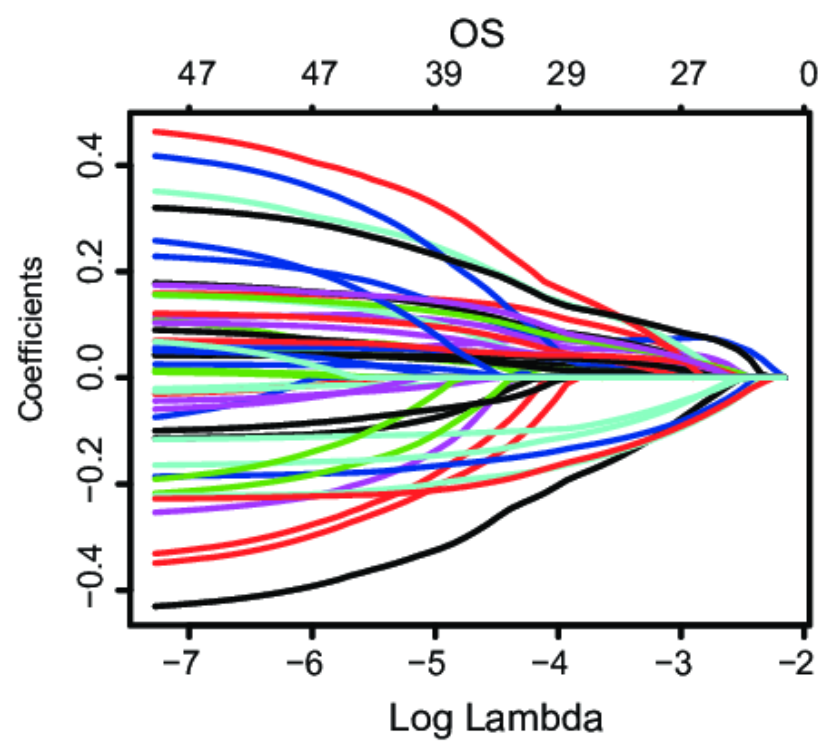

C

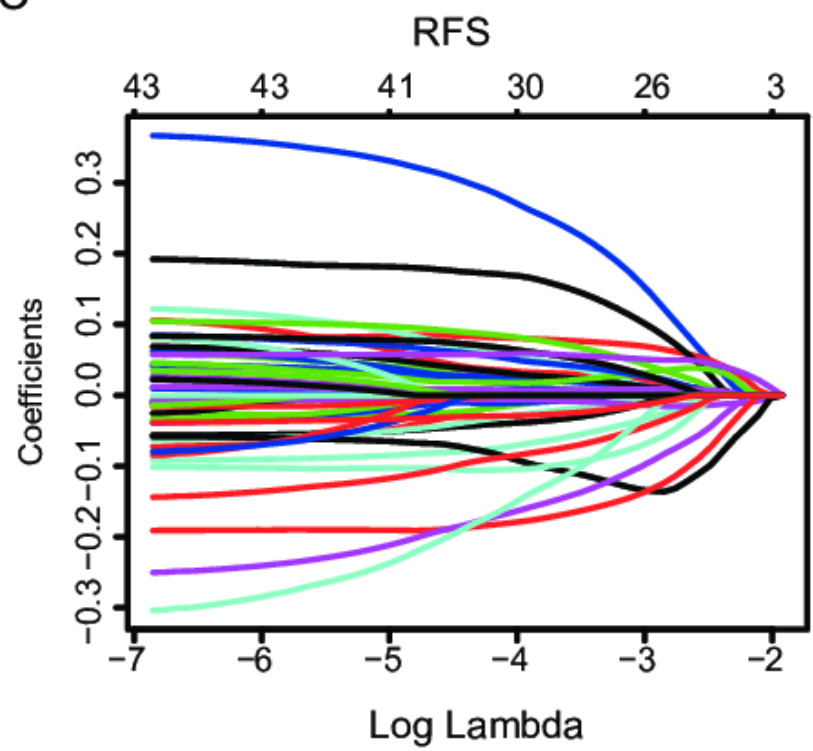

B

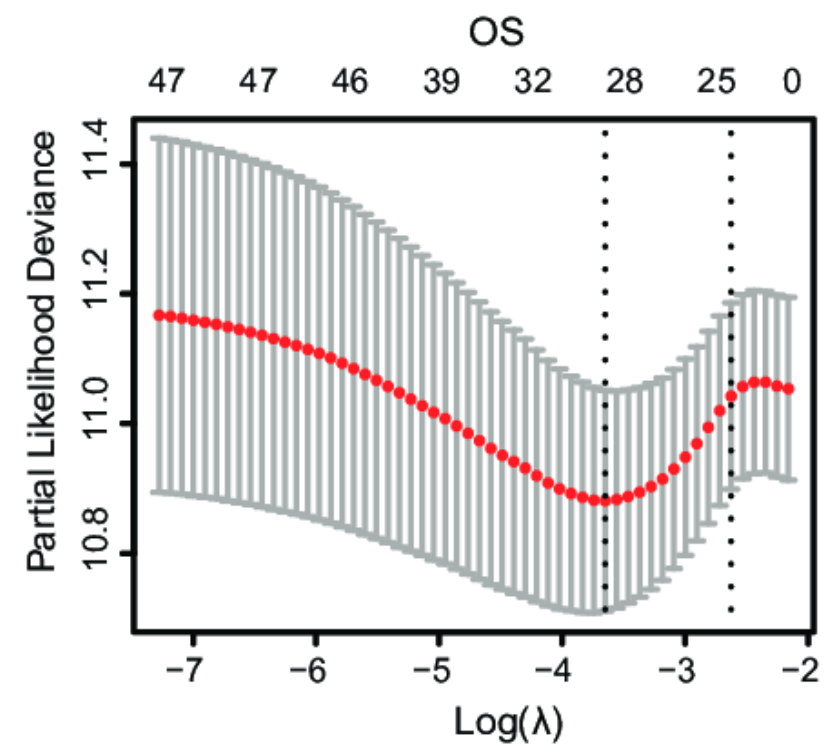

D

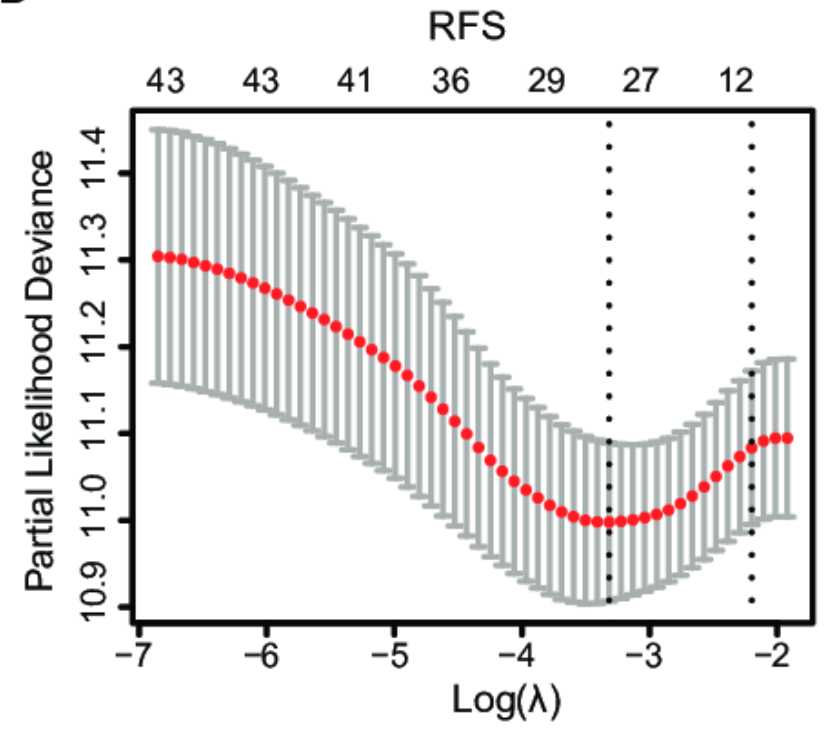


Figure 3

Establishment of gene signature for OS and RFS in TCGA dataset.

Establishment of gene signature for OS and RFS in TCGA dataset. Risk score distribution, patients' survival and status and expression heatmap of genes for OS (A) and RFS (B).

A

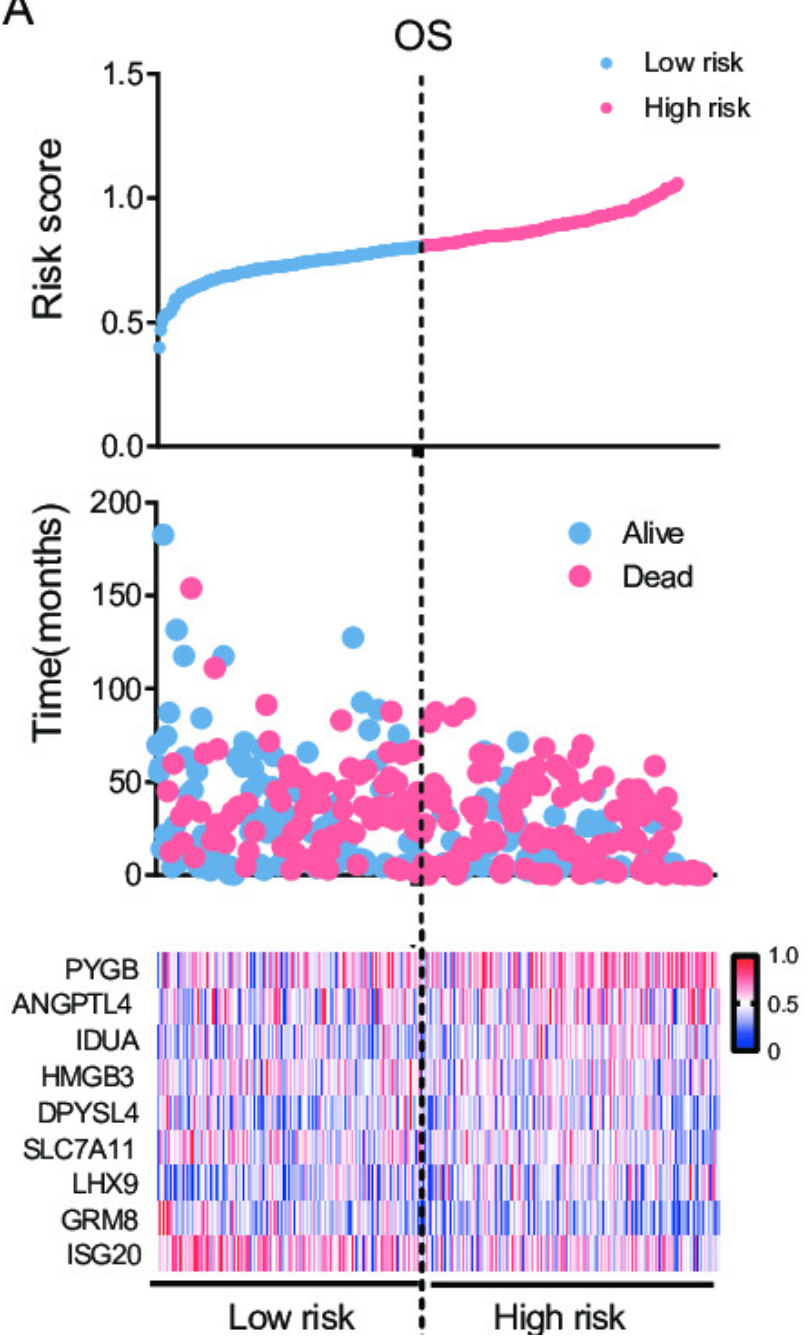

B

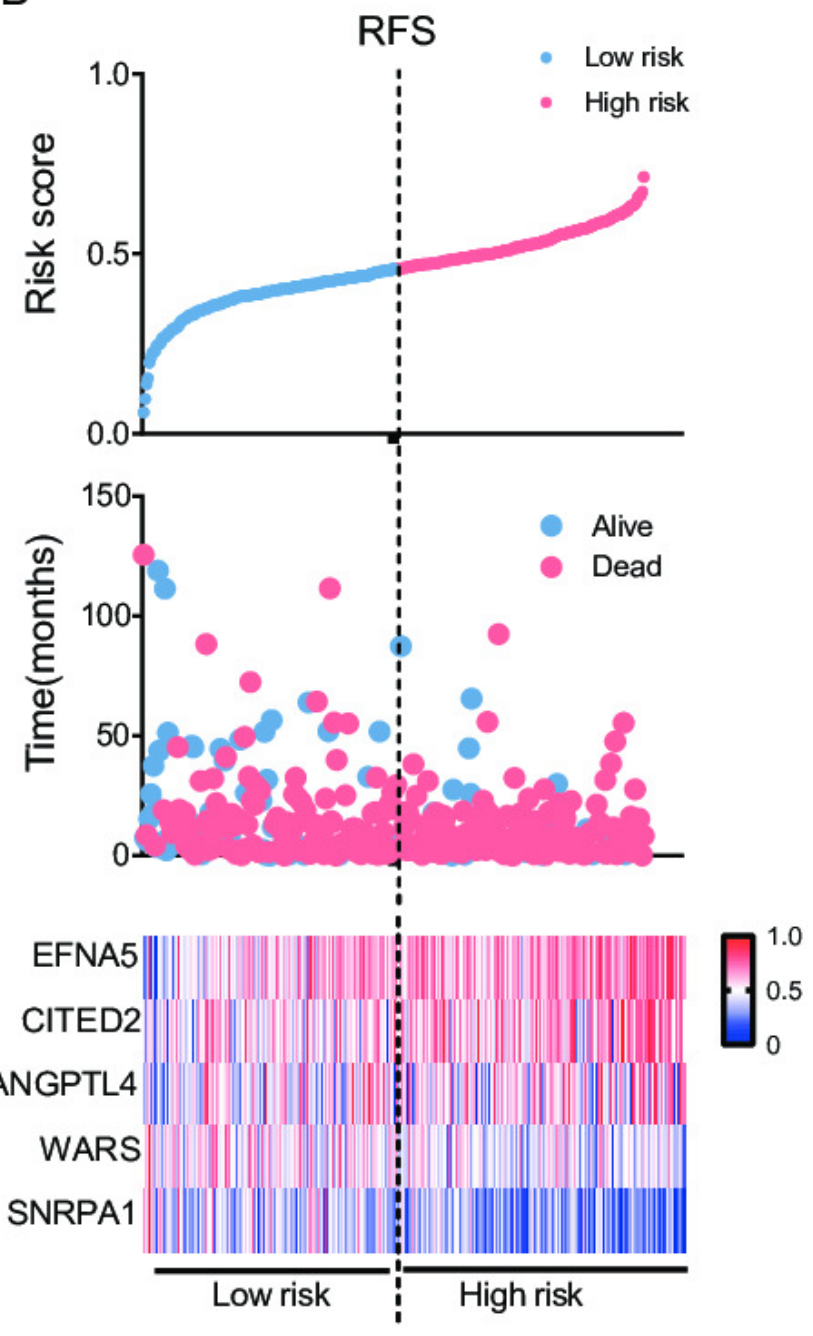


Figure 4

Kaplan-Meier curve analysis and Time-dependent ROC curve analysis

Kaplan-Meier curve analysis and Time-dependent ROC curve analysis. Kaplan-Meier curve analysis for OS (A) and RFS (B). Time-dependent ROC curve analysis of the sensitivity and specificity for OS (C) and RFS (D). 
A

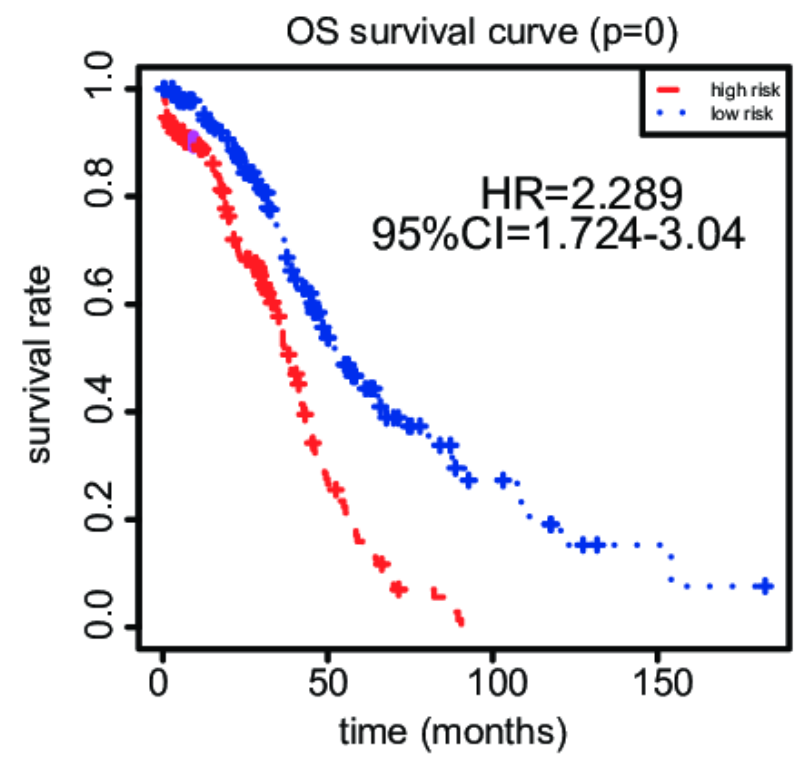

C

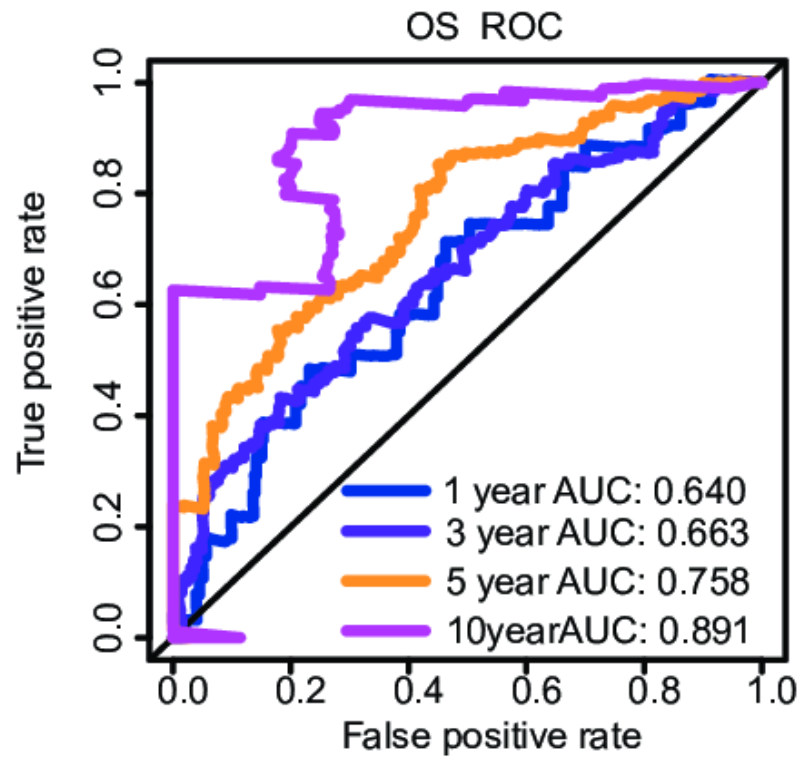

B

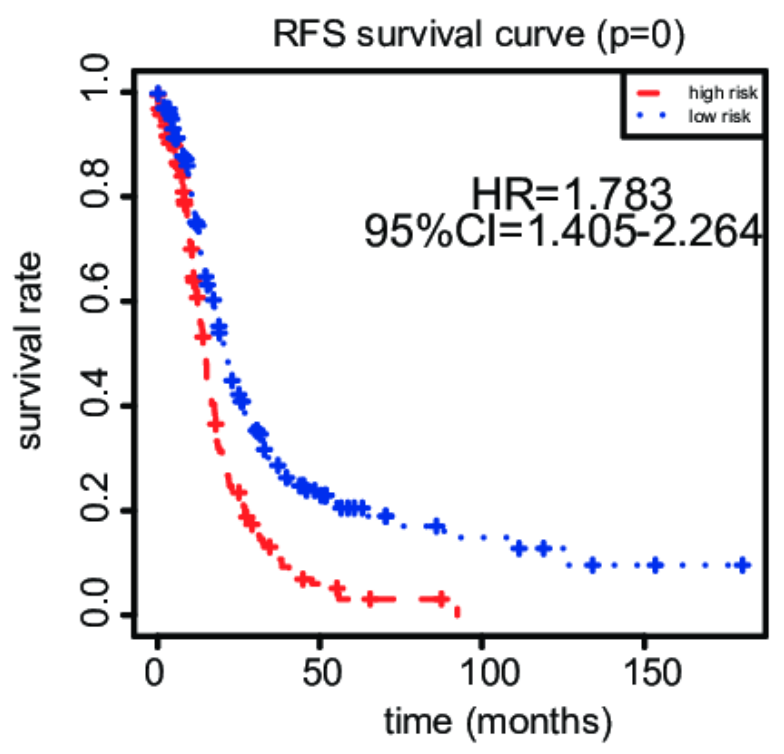

D

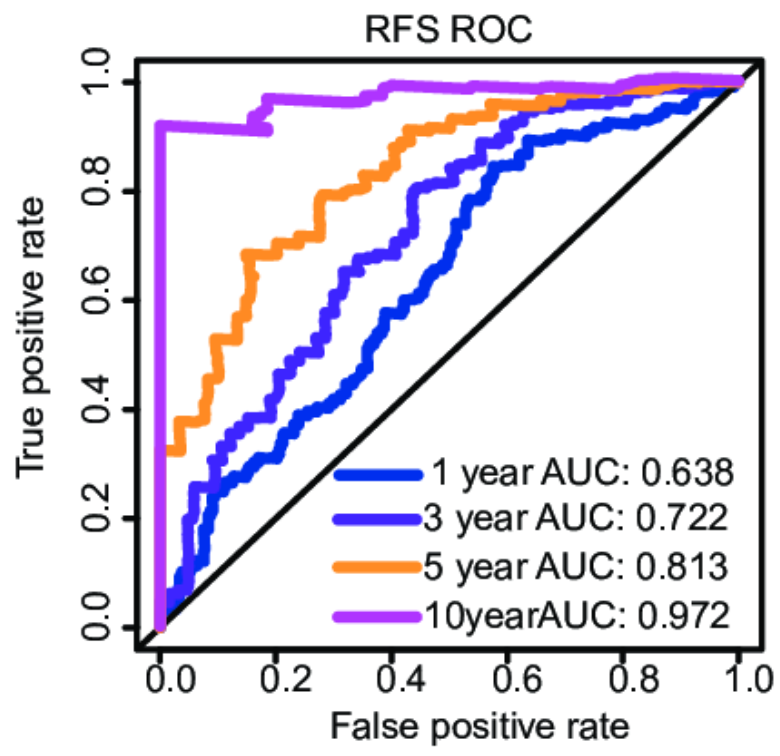


Figure 5

Kaplan-Meier curve analysis. Kaplan-Meier curve analysis

Kaplan-Meier curve analysis. Kaplan-Meier curve analysis for OS (A) and RFS (B) in GSE17260 dataset.

A

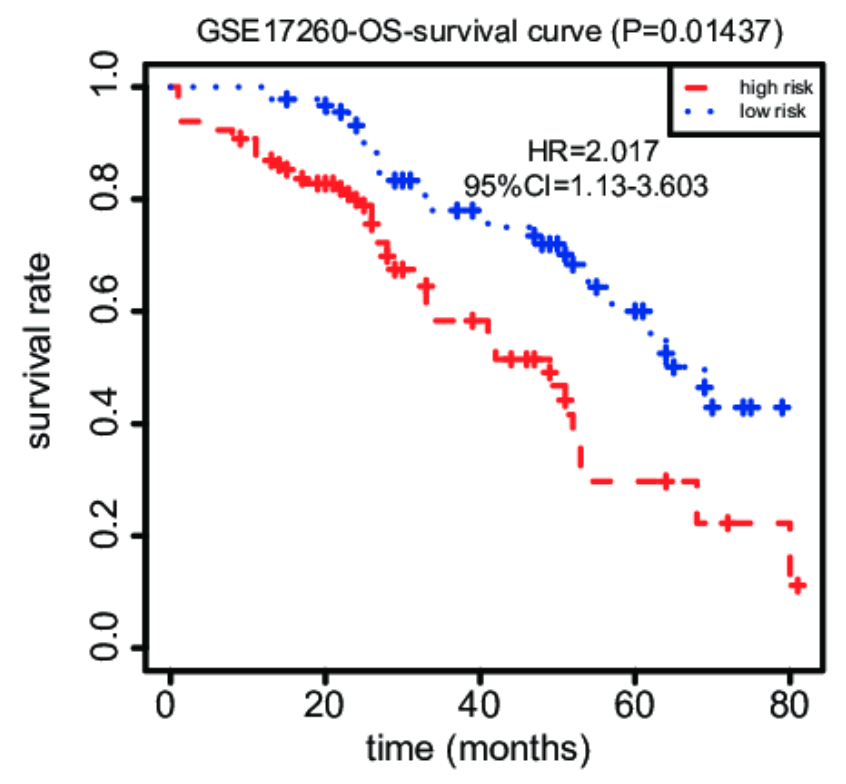

B

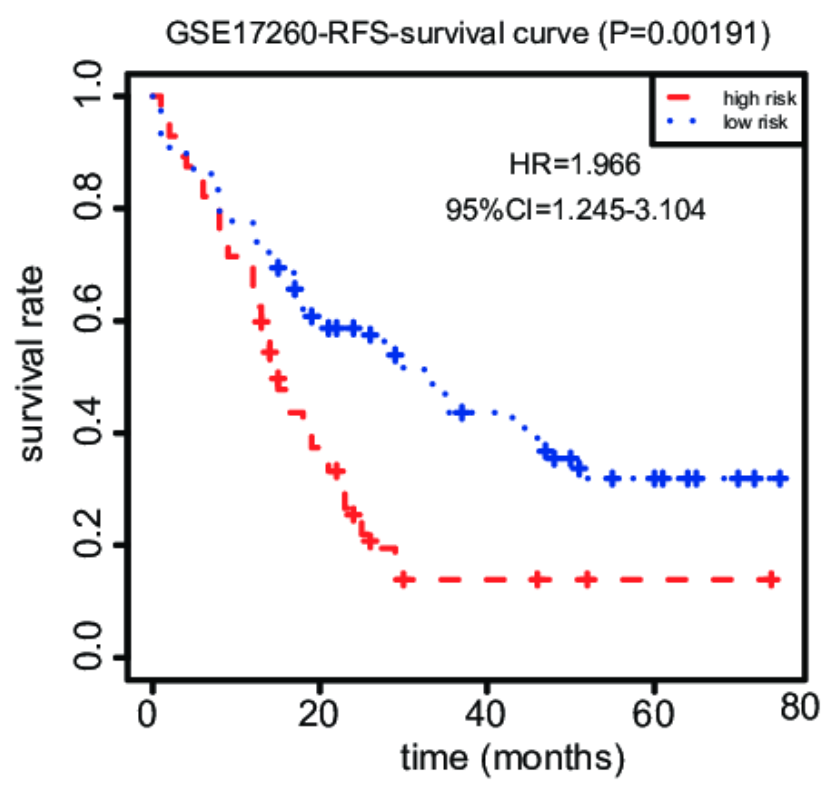


Figure 6

Prognostic value of gene signature is independent of other clinical characteristics.

Prognostic value of gene signature is independent of other clinical characteristics. Forest plot summary of univariate and multivariate Cox regression analysis of gene signature and clinical characteristics on OS (A) and RFS (B).

A

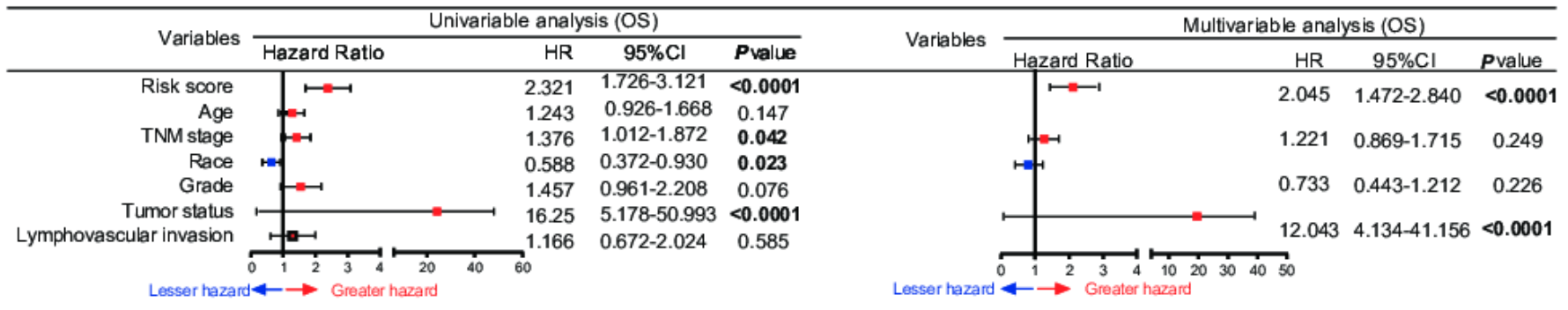

B

\begin{tabular}{|c|c|c|c|c|c|c|c|c|}
\hline \multirow{2}{*}{ Variables } & \multicolumn{4}{|c|}{ Univariable analys is (RFS) } & \multicolumn{4}{|c|}{ Multiva riable analys is (RFS) } \\
\hline & Hazard Ratio & $\mathrm{HR}$ & $95 \% \mathrm{Cl}$ & $P$ value & Hazard Ratio & $\mathrm{HR}$ & $95 \% \mathrm{CL}$ & $P$ value \\
\hline $\begin{array}{r}\text { Risk score } \\
\text { Age }\end{array}$ & $\mapsto$ & $\begin{array}{l}1.830 \\
1.140\end{array}$ & $\begin{array}{c}1.429-2.342 \\
0.884-1.470\end{array}$ & $\begin{array}{c}<0.0001 \\
0.314\end{array}$ & & 1.483 & $1.137-1.93$ & 0.004 \\
\hline TNM stage & - \pm-1 & 1.320 & $1.010-1.726$ & 0.042 & $t=-1$ & 1.163 & $0.863-1.568$ & 0.321 \\
\hline Race & & 0.872 & $0.584-1.302$ & 0.504 & & & & \\
\hline Grade & $\rightarrow-1$ & 1.339 & $0.923-1.942$ & 0.125 & & & & \\
\hline Tumor status & $\longmapsto$ & 10.706 & $6.244-18.357$ & $<0.0001$ & & 9.643 & $5.598-16.610$ & $<0.0001$ \\
\hline Lymphovascular invasion & & 1.057 & $0.678-1.649$ & 0.806 & & & & \\
\hline
\end{tabular}




\section{Figure 7}

Nomogram to predict 1-, 3- and 5-year OS and RFS for ovarian cancer patients.

The nomogram for predicting survival rate of patients with 1-, 3- and 5-year OS (A) and RFS

(C) (stage: 0 = stage I, 1 = stage II, $2=$ stage III, $3=$ stage IV; tumor status: $0=$ tumor free, $1=$ with tumor). Decision analysis curve for OS (B) and RFS (D). 
A

OS

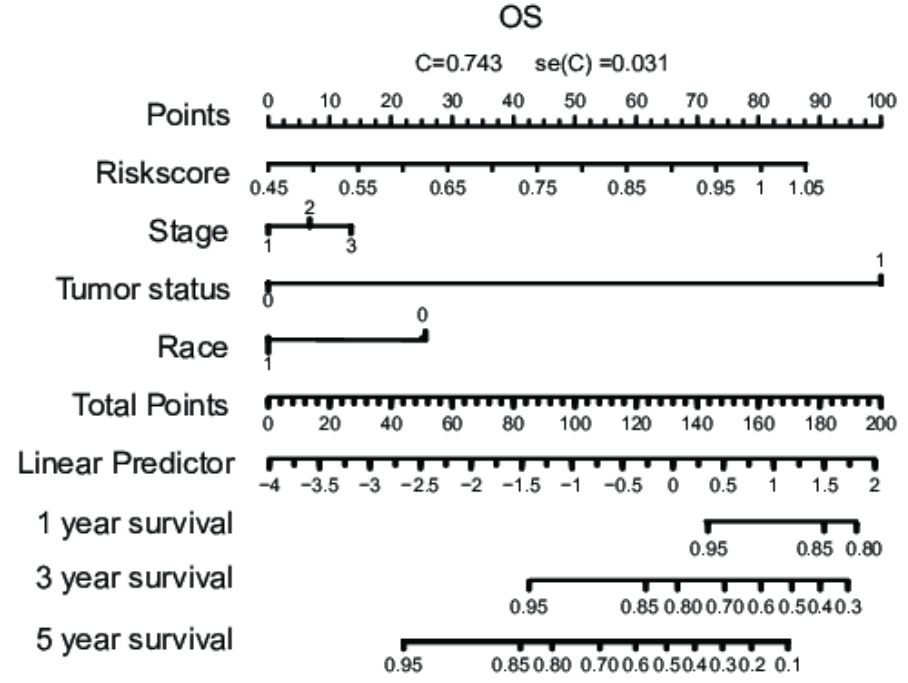

C

RFS

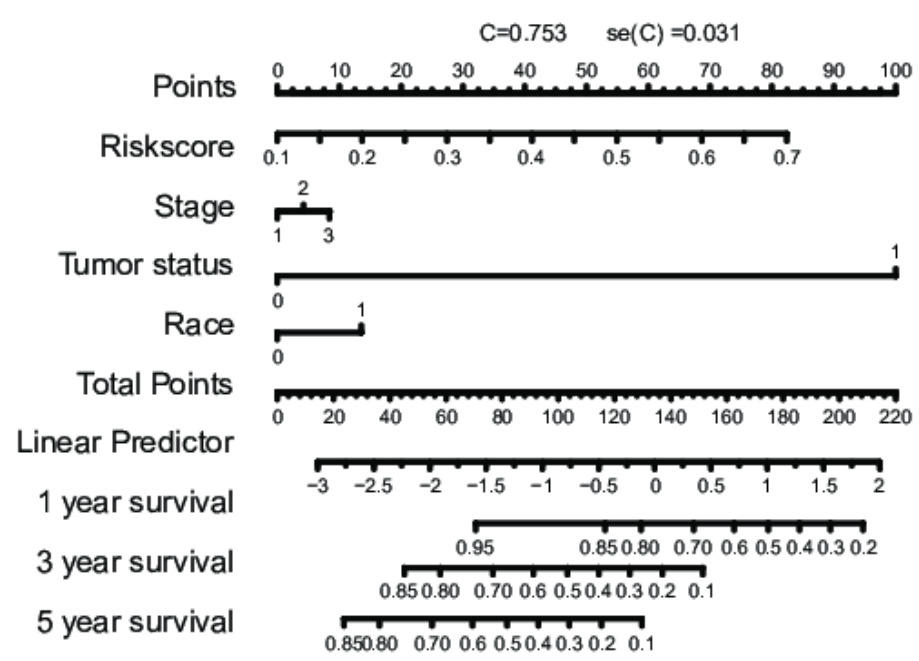

B

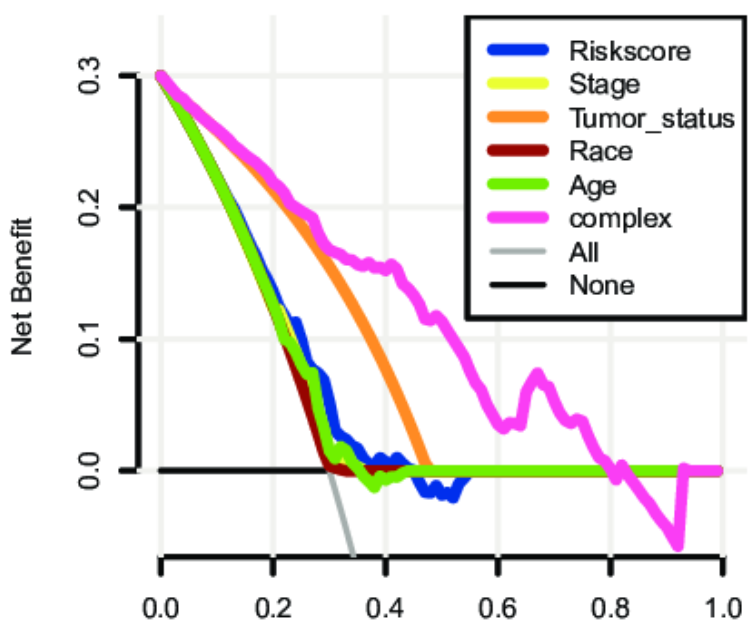

High Risk Threshold

D

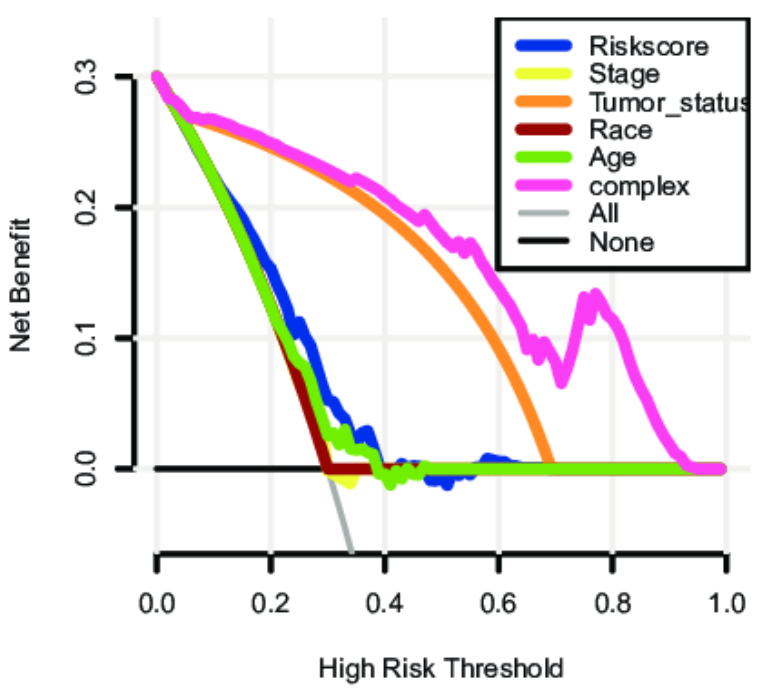

